\section{Liquid concentration mea- surement based on the optoacoustic effect and fiber Bragg grating sensor technology}

\author{
Min Rong and Lian-shui Zhang \\ Heibei University \\ College of Physics Science \& Technology \\ Baoding 071002, China
}

\begin{abstract}
An experimental system for liquid concentration measurement was set up based on the optoacoustic effect and a fiber Bragg grating (FBG) underwater sound sensor. Measurement theory and each element of the experimental setup are introduced. The light beam was injected into the liquid by a fiber bundle with a graded-refractive-index lens at the end. A matched FBG was used to demodulate the wavelength shift signals from the sensing FBG. Preliminary experimental results of sodium carbonate liquid concentration measurement were obtained, which verifies the feasibility of the method. () 2006 Society of Photo-Optical Instrumentation Engineers. [DOI: $10.1117 / 1.2189687]$
\end{abstract}

Subject terms: optoacoustic effect; fiber optic sensors; content measurement; underwater sound detection.

Paper 050706L received Sep. 10, 2005; accepted for publication Jan. 30, 2006; published online Apr. 7, 2006.

\section{Introduction}

When a laser beam is injected onto the surface of solid matter, or into a gas and liquid, an acoustic wave with a certain intensity and frequency will be created because of the interaction between laser beam and materials, and this is called the optoacoustic effect. After the 1970s, with the development of high-power lasers, more and more research has been carried out for surface measurement or component measurement of solid objects. This technology has been widely used in physics, chemistry, medicine, material science, and so on. For example, iron concentrations of composite coagulant powders were measured by means of the photoacoustic effect. A photoacoustic signal was found to be a function of the excitation power of the photoacoustic setup. ${ }^{1}$ The optoacoustic effect can also be used for gas concentration measurement. A new type of pressure sensor has been studied to improve by orders of magnitude the sensitivity of a photoacoustic measurement system using a blackbody radiation source. The pressure sensor is a cantilever-type microphone with interferometric measurement of the sensor displacement. By using a conventional filter-type photoacoustic setup with the cantilever microphone and a blackbody radiation source, we obtained a detection limit in the sub-parts-per-billion range for methane gas with a 100 -s measurement time. ${ }^{2}$ Absolute measurement of gas concentrations and saturation behavior in pulsed photoacoustics was carried out. ${ }^{3}$ The applicability of the photoacoustic principle for the detection of combustion

0091-3286/2006/\$22.00 @ 2006 SPIE gases is discussed. The use of a photoacoustic device is an additional means of detection and preventing fire and it can be realised at low cost. ${ }^{4}$ There are not many papers that discuss the application for liquid content measurement, and in almost all measurement setups, piezoelectric underwater acoustic sensors are used as the optoacoustic signal detectors. In this paper, a liquid concentration measurement based on the optoacoustic effect and fiber Bragg grating (FBG) sensor technology has been implemented. The theory and experimental setup are described in detailed. Preliminary experimental results verify the feasibility of the proposed method.

\section{Theory}

Light intensity from the YAG pulsed laser can be described as:

$I_{0}(r, t)=\frac{2 p_{0}}{\pi^{3 / 2} W_{0}^{2}} \exp \left(-k \frac{2 r^{2}}{W_{0}^{2}}-\frac{t^{2}}{\tau_{p}^{2}}\right)$,

where $I_{0}$ is the light intensity with a distance $r$ from the ray axis, $E=\tau_{p} \cdot p_{0}$ is the laser pulse energy, $\tau_{p}$ is the time width when the laser pulse intensity becomes as small as $1 / e$ of the maximum intensity, and $W_{0}$ is the laser beam width.

When the pulsed laser is injected into the liquid, the laser energy is absorbed and heat is released from the liquid. Thus, a heat source comes into being as:

$S_{t}(r, t)=\frac{\partial I_{A}}{\partial t}=k(1+c) \frac{\partial I_{0}}{\partial t}$,

where $I_{A}=k(1+c) I_{0}$ is the absorbed light intensity by the liquid, $c$ is the liquid concentration, $k=a_{0} b$ is a constant, $a_{0}$ is the absorption coefficient, and $b$ is the medium thickness laser beam passing through.

The elastic wave $p$ created by the heat source can be solved by the following wave equation:

$$
\left(\frac{1}{v^{2}} \frac{\partial^{2}}{\partial t^{2}}-\nabla^{2}\right) p(r, t)=\frac{\beta}{C_{p}} S_{t}(r, t),
$$

where $v$ is the sound velocity in liquid, $\beta$ is the isothermal expansion coefficient in liquid, and $C_{p}$ is the specific heat at constant pressure.

By substituting Eq. (2), Eq. (3) can be rewritten as:

$\left(\frac{1}{v^{2}} \frac{\partial^{2}}{\partial t^{2}}-\nabla^{2}\right) p(r, t)=\frac{\beta}{C_{p}} k(1+c) \frac{\partial I_{0}}{\partial t}$.

By substituting Eq. (1), Eq. (4) can be rewritten as:

$$
\left(\frac{1}{v^{2}} \frac{\partial^{2}}{\partial t^{2}}-\nabla^{2}\right) p(r, t)=\frac{2 \beta k(1+c) E}{\pi^{3 / 2} W_{0}^{2} \tau_{p} C_{p}} \exp \left(-k \frac{2 r^{2}}{W_{0}^{2}}-\frac{t^{2}}{\tau_{p}^{2}}\right) .
$$

If only one dimension is considered in the measurement setup, intensity of the optoacoustic signal can be deduced $\mathrm{as}^{5}$ :

$p=\frac{E \beta}{(2 \pi \varepsilon)^{3 / 2} C_{p}}\left|\frac{v}{r}\right|^{1 / 2} k(1+c)=\frac{E \beta}{(2 \pi \varepsilon)^{3 / 2} C_{p} t^{1 / 2}} k(1+c)$,

where $\varepsilon=\left[\tau_{p}^{2}+\left(W_{0}^{2} / 2 v^{2}\right)\right]^{1 / 2}$. Because Beer's law is suitable for the weak solution, the variation of $v^{1 / 2}$ can be ignored 


\section{OE LETTERS}

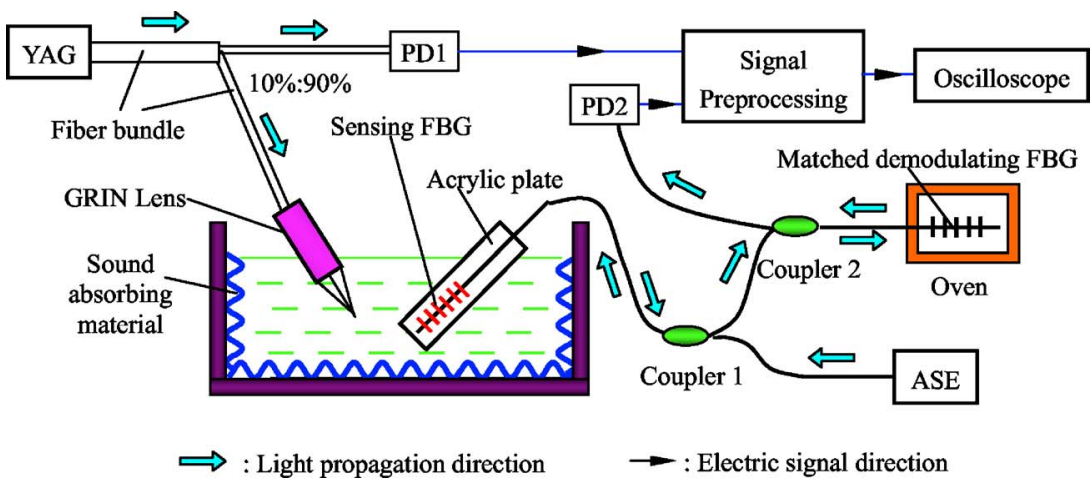

Fig. 1 Experimental setup for liquid concentration measurement based on optoacoustic effect and FBG sensor.

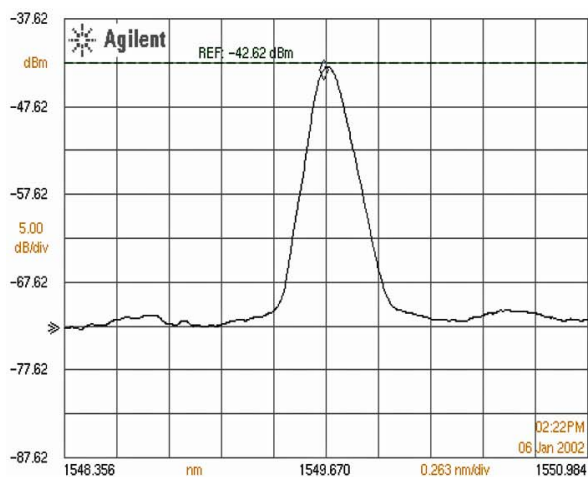

(a) $\mathrm{c}=0$

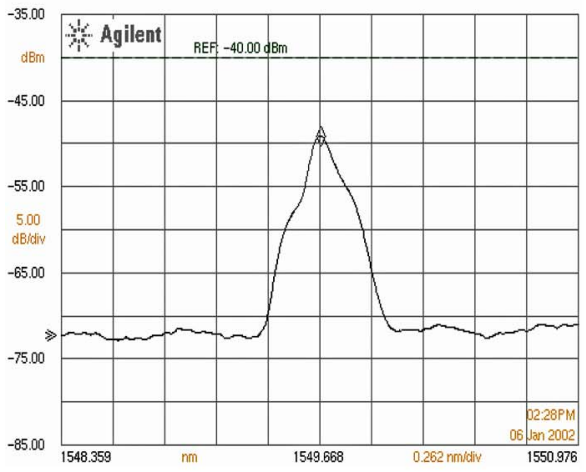

(a) $\mathrm{c}=2 \%$

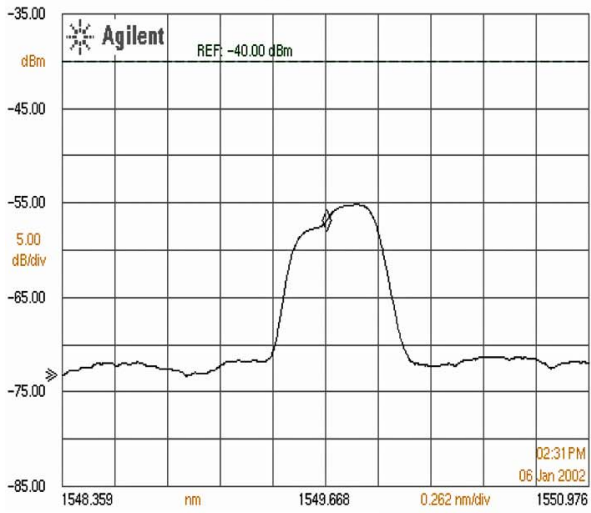

(a) $\mathrm{c}=5 \%$

Fig. 2 Spectrograms recorded by OSA as the increment of sodium carbonate mass percent changes, where $c$ represents liquid concentration. when the liquid concentration is small. In the measurement process, with different concentration, the measuring point is invariable, that is, $r$ is a constant, so $t^{1 / 2}=|r / v|^{1 / 2}$ can also be regarded as a constant. Thus, from Eq. (6), we can see that there exists a linear relation between the sound intensity and the liquid concentration.

\section{Experimental Setup and Preliminary Results}

The experimental setup is shown in Fig. 1. A YAG pulsed laser with an output power of $1 \mathrm{~W}$, an output wavelength of $1.06 \mu \mathrm{m}$, a pulse width of $10 \mathrm{~ns}$, and a modulation frequency of $22 \mathrm{~Hz}$ is used as the exciting light source. The laser pulse is coupled into the multimode fiber bundle, $10 \%$ of which is directly detected by a photodiode (PD1) as the reference signal, and other light is coupled into the emission probe, which has a graded-refractive-index (GRIN) lens at the end. The reference signal received by PDl is useful for eliminating the effect on the measurement accuracy caused by fluctuation of the light source. The GRIN lens acts as a converging lens to focus the exiting light.

The optoacoustic tank is made of polymethyl methacrylate with a length of $100 \mathrm{~cm}$, a width of $6 \mathrm{~cm}$, and a height of $40 \mathrm{~cm}$. To reduce the noise interference from sound reflection caused by the tank wall, wedge-shaped sound absorbing material is used in the bottom and around the tank wall.

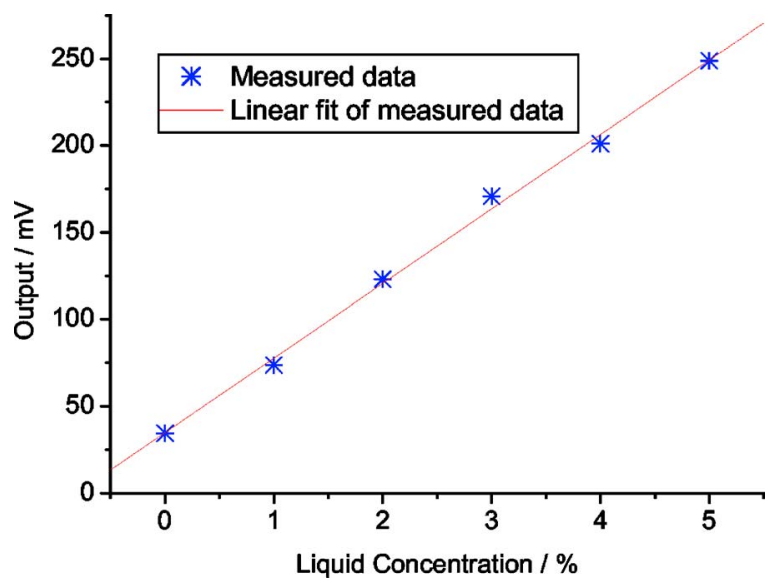

Fig. 3 Calibration curve of the liquid concentration measurements. 
The center of the sensor probe for detecting the sound signal is placed $25 \mathrm{~cm}$ aside the optoacoustic action area (focus point of the GRIN lens). The sensor probe is a FBG, bonding on the surface of an acrylic plate. The acrylic plate is used as a support and because of its good elastic characteristic, it can improve the pressure sensitivity of the FBG, which is very useful for the liquid concentration measurement. The FBG used in the sensor probe is made of common single-mode fiber with center wavelengths of $1550.232 \mathrm{~nm}$, a $3-\mathrm{dB}$ bandwidth of $0.195 \mathrm{~nm}$, and a reflectivity of $98.8 \%$.

A simple matched FBG wavelength shift demodulation method is used in this system. ${ }^{6}$ The matched demodulating FBG is also made of common single-mode fiber with center wavelengths of $1550.230 \mathrm{~nm}$, a 3-dB bandwidth of $0.194 \mathrm{~nm}$, and a reflectivity of $98.1 \%$. It is placed in a constant-temperature oven and free of strain to keep the center wavelength invariable.

Experiments for sodium carbonate liquid concentration measurement were carried out. First, the spectrograms were observed and recorded by an optical spectrum analyzer (OSA) at the point of PD2 in Fig. 1 during the process of changing the increment of sodium carbonate mass percent. Figure 2 shows the results, from which we can see that as the increment of sodium carbonate mass percent changes, the two matched reflection spectra separate from each other, and the maximum light intensity drops obviously.

PD2 was used to detect the light intensity instead of the OSA, and measurement characteristics of the sodium carbonate liquid concentration based on the optoacoustic effect and FBG sound sensor were calibrated, as shown in Fig. 3, from which we can see that there is a good linear relation between the liquid concentration and the measurement output. This linear measurement characteristic agrees well with the theoretical analysis.

\section{Conclusions}

Liquid concentration measurement based on the optoacoustic effect and FBG sensor technology is proposed, and the experimental setup is introduced in detail. It can be concluded that the advantages of this method are:

- the FBG sound sensor has better sensitivity compared with piezoelectric sound sensors;

- it can be used in acidic, alkaline, or corrosive liquid;

- it can be used in liquids with high temperature;

- it has a faster response time; and

- it is very easy to implement a distributed measurement.

\section{References}

1. M. Fan, R. Brown, and S.-W. Sung, "Photoacoustic measurement of iron in composite coagulant," Chem. Eng. Process. 42, 553-559 (2003).

2. J. Kauppinen, K. Wilcken, I. Kauppinen, and V. Koskinen, "High sensitivity in gas analysis with photoacoustic detection," Microchem. J. 76, 151-159 (2004)

3. S. Schafer, A. Miklos, A. Pusel, and P. Hess, "Absolute measurement of gas concentrations and saturation behavior in pulsed photoacoustics," Chem. Phys. Lett. 285, 235-239 (1998).

4. P. W. Nebiker and R. E. Pleisch, "Photoacoustic gas detection for fire warning," Fire Saf. J. 36, 173-180 (2001).

5. Q. C. Wang, M. C. Huang, and H. W. Sun, "Optoacousitic deflection technology excitated by pulsed laser in liquid," Acta Acust. 15(1), 53-59 (1990) (in Chinese).

6. Y. Zhao, J. Yang, B. J. Peng, and S. Y. Yang, "Experimental research on a novel fiber-optic cantilever-type inclinometer," Opt. Laser Technol. 37(7), 555-559 (2005). 